\title{
Impact of Social Interactions on Demand Curves for Innovative Products
}

\author{
K. Maciejowska*, A. Jędrzejewski, A. Kowalska-Pyzalska and R. Weron \\ Department of Operations Research, Wrocław University of Technology, Wrocław, Poland
}

\begin{abstract}
Empirical studies suggest that word-of-mouth strongly influences the innovation diffusion process and is responsible for the "S" shape of the adoption curve. However, it is not clear how word-of-mouth affects demand curves for innovative products and strategic decisions of producers. Using an agent-based model of innovation diffusion, which links consumer opinions with reservation prices, we show that a relatively strong word-of-mouth effect can lead to the creation of two separated price-quantity regimes, with a nonlinear transition between them. A small shift of the product's market price can result in a drastic change of the demanded quantity and, hence, the revenues of a firm. Using Monte Carlo simulations and mean-field treatment we demonstrate that word-of-mouth may have ambiguous consequences and should be taken into account when designing marketing strategies.
\end{abstract}

DOI: 10.12693/APhysPolA.129.1045

PACS/topics: 89.65.Gh, 87.23.Ge

\section{Introduction}

The ways and conditions influencing a successful diffusion of a new product are of particular interest to economists and analysts who design marketing strategies [1-3]. Since it is either very costly or - in many cases - impossible to conduct field experiments regarding an innovation's diffusion in the market, there are only two plausible investigation approaches: collecting ex post the information on the diffusion or designing ex ante a model and performing simulations and/or analytical calculations. In the latter case, investigators typically either adopt the simplistic framework of the now-classical Bass model or develop a tailor-cut agent-based model that allows for (generally) realistic simulations of the diffusion process [4].

In this paper we place ourselves within the simulationbased ex ante approach. We utilize a relatively simple, yet sufficiently rich agent-based model developed recently by Kowalska-Pyzalska et al. [5], which links the opinions of potential consumers ${ }^{\dagger}$ with their market behavior via the concept of the reservation price $-P_{i}$, i.e. the highest price that agent $i$ will accept and still purchase the good [7]. We conduct the study from the supply point of view and aim at providing a producer (a seller) of an innovative good with advice on pricing and marketing strategies. We assume that the producer operates in a noncompetitive market and hence is able to set the market price of the product, $P \in[0,1]$, at any desired level. Note that the noncompetitiveness refers to the market

\footnotetext{
* corresponding author; e-mail: katarzyna.maciejowska@pwr.edu.pl

†In the text, we use the words "consumer", "agent" and "individual" interchangeably with spinson, a term coined by Nyczka and Sznajd-Weron [6] that nicely allows to go around gender issues and reflects the dyadic nature of an agent's opinion $(\rightarrow$ spin $)$ and the object of study ( $\rightarrow$ person).
}

structure (there is just one producer or seller) and does not restrict the number of products available in the market in any way. In fact, the prices we consider $-P$ and $P_{i}$ - are in relative terms (not nominal) and relate the price of the innovation to the prices of existing goods of similar nature. In order to comply with the commonly accepted standard in the economic literature, we present the results in terms of the demand curve, which considers the market price of the product as a function of the quantity sold, $P(Q)$. However, we derive the demand curve from the penetration rate or level $[2,3,5], Q(\cdot)$, i.e. the percentage of adopted consumers as a function of model parameters (in particular, of market price $P$ ).

Using Monte Carlo simulations (for artificial and real social networks from Facebook and Google+) and meanfield (semi-)analytical treatment (for fully connected networks, i.e. complete graphs), we study the influence of three micro (i.e. consumer) level components on the market demand curve: (i) individual judgment, (ii) social influence (i.e. word-of-mouth, WOM) and (iii) mass-media influence. By individual (or personal) judgment we understood the evaluation of the product according to its non-financial costs and benefits, as well as the perception of the behavioral difficulty connected with its adoption. We assume that personal judgment is neither affected by mass-media nor by WOM, hence it is closely related to the concept of independent behavior [8-10]. In the model it occurs with (independence) probability $p \in[0,1]$. Although innovative products are assumed to be beneficial, it is possible that a spinson will reject it if the potential behavioral costs outweigh the gains. In order to capture the adoption difficulty [11], the probability of a negative product evaluation is described by parameter $f \in[0,1]$. On the other hand, with probability $(1-f)$ the spinson will evaluate the product positively, because the benefits of adopting the innovation exceed its non-financial costs.

Word-of-mouth (WOM) is defined as informal advice passed between agents, i.e. a form of social (or internal) influence. It has been found to have significant impact on 
consumer behavior and innovation diffusion [2, 12, 15]. In the adopted here so-called situation approach [16], each spinson behaves independently with probability $p$ and with probability $(1-p)$ is exposed to WOM from a group of $q$ neighboring (i.e. directly connected; in this study we use $q=4$, for a discussion see [5]) spinsons or advertising. When a spinson is confronted by a unanimous group of $q$ neighboring spinsons sharing the opposite opinion, it will conform to peer pressure [10] and choose a new reservation price (resulting in opinion change). However, if at least one of the $q$ neighbors shares the opinion of the spinson, WOM will have no effect on the reservation price and the spinson will be exposed to advertising, i.e. an external influence. The impact of advertising is expressed by parameter $h \in[0,1]$, the probability that a spinson increases the product evaluation due to new information obtained from mass media.

The contribution of this paper is twofold. Firstly, using a relatively simple, yet sufficiently rich agent-based model of opinion formation we show that a strong WOM effect can lead to the creation of two separated price-quantity regimes, with a nonlinear transition between them. Even a small shift of the market price can result in a drastic change of the demanded quantity and, hence, the revenues of a firm. Moreover, strong WOM combined with behavioral difficulty of adoption may hamper the diffusion process and substantially reduce the demand even for very low prices. Importantly, this result is obtained both for fully connected networks, which can represent cliques in the society, as well as sample Facebook and Google + social networks of variable sizes and characteristics. Secondly, the results show that WOM may have an ambiguous impact on the diffusion process, leading to either an increase or a decrease of the total demand depending on the system characteristics and the market price. This observation substantially expands the findings of $[14,15]$, that revealed only the negative influence of WOM on innovation diffusion.

\section{Economics of innovation diffusion}

To the best of our knowledge, only a few authors have used agent-based modeling for constructing the demand curve and deriving market prices. In particular, Campbell [14] models WOM as a percolation process on the social network and assumes that agents engage in WOM with a probability, which is a function of the agent's valuation of the good and the market price. Only these agents who are willing to purchase the product are prepared to pass on the information about it to others. The paper concludes that demand is more elastic and prices are lower under WOM. Cantono and Silverberg [17] observe that diffusion can reduce the market price. In their model, the price becomes a function of the number of previously adopted agents. Hohnisch et al. [18] find that diffusion of new products can be delayed by a drift of the percolation dynamics from a non-percolating regime to a percolating regime, which occurs because the probability of buying increases over time with the cumulative number of buyers. In their article, the heterogeneity of reservation prices plays a central role and determines whether diffusion takes place or not. Finally, Zeppini and Frenken [15] show a percolation critical transition from a diffusion to a no-diffusion regime, with a price threshold value depending on the network structure.

In these papers the diffusion of innovation is conditioned on information spreading and the authors do not consider the potential influence of the opinion formation mechanism. However, the infection-spreading models, such as percolation and generalized contagion processes, are not consistent with the experimental data [19]. On the contrary, approaches utilizing opinion dynamics are able to reproduce the empirical data and are suitable for innovation diffusion modeling. In this study we take the latter findings as a staring point and use an opinion formation model proposed by Kowalska-Pyzalska et al. [5] to investigate how the micro-level social interactions influence the shape of the demand curve for innovative products and producer decisions. By doing so, we fill the existing gap and contribute to the literature on innovation diffusion.

\section{Results}

To study the influence of WOM, advertising and personal judgment on the demand curve we perform Monte Carlo simulations (for artificial and real social networks from Facebook and Google+) and a mean-field analysis (for complete graphs, CG). The Monte Carlo simulations are conducted for systems of variable sizes, ranging from $N=338$ to 4289 spinsons for Facebook and Google+ networks and from $10^{2}$ to $10^{4}$ spinsons for CG (only the results for the largest $\mathrm{CG}$ are plotted later in the text). All simulation results are averaged over $10^{4}$ samples (or trajectories; not to be confused with the number of spinsons, i.e. $N$ ). We use a random sequential updating scheme with the time measured in Monte Carlo steps (MCS). Namely, one MCS consists of $N$ elementary time steps $\Delta t$ in which one spinson is randomly selected and undergoes the procedure visualized in Fig. 1 in [5]. To consider only the stationary states of the system we collect the data after $10^{3}$ MCS.

The mean-field approach (MFA) used here assumes that the network is homogeneous (in the sense that the local concentration of adopted agents is statistically equal to the global, system-wide concentration) and of infinite size (i.e. $N=\infty$ ). However, even for complete graphs as small as 100 spinsons the mean-field and simulation results coincide very well. For derivation and form of the MFA formulae we refer to Eqs. (1)-(3) in [5].

\subsection{Results for complete graphs}

We use a simplified model without WOM as the benchmark for the analysis of social influence effects. In this model there are only two factors influencing reservation prices: individual judgment and advertisement. With probability $p$ a spinson will behave independently and with probability $(1-p)$ will be exposed to the massmedia influence. In the latter case, with probability $h$ the spinson will increase its reservation price. 
In the absence of WOM, there are two major factors - the adoption difficulty and the mass-media influence - which shift the demand curve in opposite directions. When $f=h=0$ then the demand is a linear function: $P(Q)=1-Q$, where $Q$ is the penetration rate. Generally, for models without the mass-media effect, it can be easily shown that $P(Q)=1-Q /(1-f)$, compare the top left and right parts in Fig. 1. For models with advertising, i.e. with $h>0$, the demand curve is always concave, see the bottom right part in Fig. 1. It does not have a closed, analytic form and therefore needs to be calculated numerically.
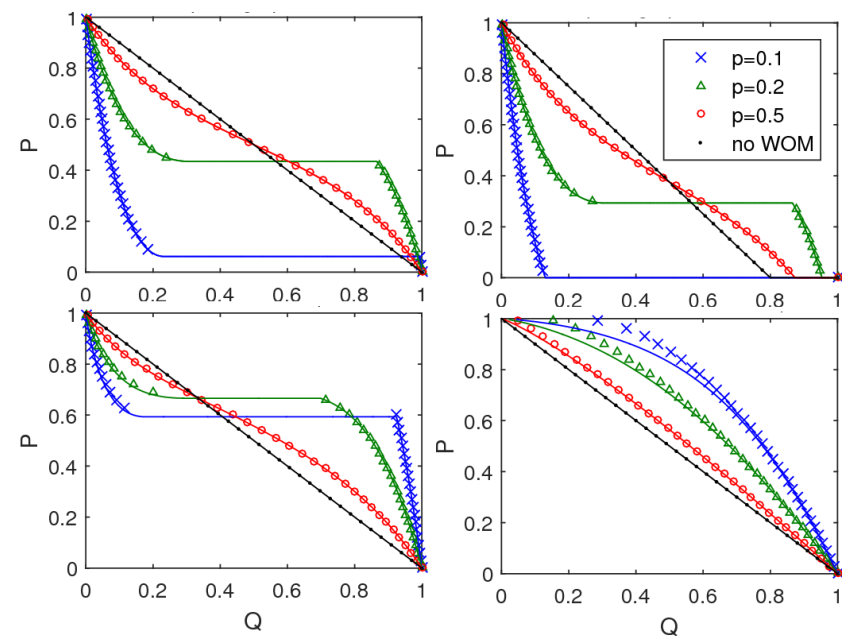

Fig. 1. Demand curves $P(Q)$ for models with different independence probabilities $(p=0.1,0.2,0.5)$ : without the difficulty effect and advertising $(f=h=0$; top left), with the difficulty effect but without advertising ( $f=0.2, h=0$; top right) and without the difficulty effect but with advertising ( $f=0, h=0.2$; bottom left). For comparison, the results for the benchmark models without WOM are also plotted (bottom right). Note, that for the latter the mean-field approximation [5] does not work that well for high $P$. Monte Carlo simulation results for a complete graph consisting of $N=10^{4}$ spinsons are denoted by symbols, whereas the corresponding mean-field results for an infinite system by solid lines of the same color.

Now let us consider the general model with WOM, as proposed in [5], but without the difficulty effect and advertising. When $f=h=0$, the strength of WOM which increases with probability $(1-p)$ - has a substantial effect on the demand curve. For high market prices it significantly reduces the demand quantity, whereas for low market prices it boosts the consumption, see the top left part in Fig. 1. These results expand the findings of $[2,15]$, who have concentrated only on the negative effects of WOM.

Moreover, it can be observed that for low values of independence probability $p$, there are regions characterized by a very high demand elasticity. For example, for $p=0.1$ and market prices around $P=0.063$, a small change of the market price may result in a substantial change of the market penetration level. For $P=0.065$, the demand is $Q=0.22$, whereas for 0.063 it rises to 0.99 ! Hence a decrease in the market price by 0.02 leads to a huge demand increase of 0.77 (on a $[0,1]$ scale). As a result, two price regimes can be identified: high price/low adoption and low price/high adoption, with a nonlinear transition between them. The switch is the more rapid, the lower is the personal judgment effect, i.e. probability $p$, and the stronger the WOM effect. This diffusion feature has an important implication for producers, who need to take into account the shape of the demand curve when setting the market price of the product. A small overestimation of the price could block the diffusion and lead to large revenue losses.

It should be emphasized that a similar pattern has been found in [15] within a percolation framework. The main difference between our studies comes from the fact that in [15] an (almost) full market penetration is obtained only for prices close to zero. On the contrary, our results show that a high adoption regime (with the market penetration of around 90\%) could be also reached for moderate prices, e.g. for $p=0.2$ and $P=0.4$.

In order to isolate the difficulty effect, the demand curves for $f=0.2$ and $h=0$ are plotted in the top right part of Fig. 1. In the case of weak WOM, i.e. $p=0.5$, the demand curve does not differ significantly from the benchmark, no WOM model. The adoption difficulty reduces, but does not stop the diffusion. A totally different picture arises when a model with strong WOM, i.e. $p=0.1$, is considered. WOM combined with the adoption difficulty blocks the diffusion, which reaches only $Q=0.133$, even for a market price very close to zero! This result may help to explain, why some of the innovations do not succeed and never reach a satisfactory market penetration level. It provides a warning for companies, which propose a new but difficult to adopt technology. Regardless of the potential benefits, WOM may stop the diffusion process. In such a case, producers should aim at weakening the WOM effect by properly designed marketing campaigns. When models with moderate WOM (i.e. $p=0.2,0.3$ ) are analyzed, conditional on the level of the market price, WOM may either increase or decrease the effect of the adoption difficulty. The largest differences occur around the transition price, which marks the threshold separating full market penetration from a low penetration regime. Finally, to better illustrate the difficulty effect in the left part of Fig. 2 we plot demand curves for the same level of independence and advertising $(p=0.1, h=0.2)$ but six different levels of difficulty $(f=0,0.1, \ldots, 0.5)$.

In the considered model, advertising is assumed to have a positive impact on the diffusion process and the demand. In order to focus on this phenomenon, we now consider models with no difficulty effect, i.e. with $f=0$. First, it should be noticed that parameter $p$ affects both the advertisement and WOM effects. In the case of no WOM, the lower the independence level $p$, the stronger the mass-media influence, see the bottom right 

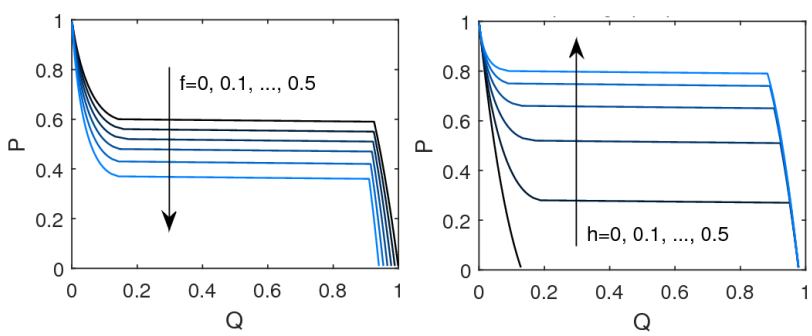

Fig. 2. Dependence of demand curves $P(Q)$ on the difficulty effect (left part) and advertising (right part) for the same level of independence $p=0.1$. Only meanfield results for an infinite system are plotted. Arrows indicate the direction of changes in $f$ or $h$, from 0 to 0.5 with $\Delta f=\Delta h=0.1$.

part in Fig. 1. Hence, small values of $p$ support the diffusion. On the contrary, when WOM is allowed, a low level of $p$ increases the social pressure and may slow down the diffusion, see the bottom left part in Fig. 1. It can be observed that WOM significantly changes the shape of the curve and the potential penetration levels. Similar to the $f=h=0$ case, the differences are more significant for smaller independence probabilities $p$. Moreover, as in the previously studied cases, the influence is not uniform across the different market prices. For a high market price, WOM reduces the effect of advertising, whereas for a low price it supports the diffusion. For instance, for $p=0.2$ WOM reduces the demand by 0.361 when the market price is $P=0.7$, but when $P=0.6$ it increases the demand by 0.182 . Finally, to better illustrate the effect of advertising in the right part of Fig. 2 we plot demand curves for the same level of independence and difficulty $(p=0.1, f=0.2)$ but six different levels of advertising $(h=0,0.1, \ldots, 0.5)$.

\subsection{Results for sample Facebook and Google+ social networks}

Up to this point we have considered artificial, fully connected networks. Although complete graphs can be used to study cliques in the society, in general, they are not suitable for modeling real social networks. Hence, now we present the results for sample Facebook and Google+ networks. Due to space limitations we consider only four networks (see Table I for their descriptive statistics) and only one model type (with the difficulty effect, $f=0.2$, but without advertising, $h=0$; as in the top right part in Fig. 1). However, for other analyzed social networks and model types the results were qualitatively the same when compared with the results obtained for complete graphs and corresponding models.

The network structures were downloaded from the SNAP database (see http://snap.stanford.edu/ data). The four selected networks, two from Facebook and two Google+, vary in size and other network characteristics. Nevertheless, the general picture is very similar to the one for the complete graphs, see Fig. 3. Namely, a strong WOM effect can lead to the creation of two separated price-quantity regimes, with a nonlinear transition
TABLE I

Descriptive statistics of the four social networks considered in this study. The first two are "circles" (or "friends lists") from Facebook, the last two are "circles" from Google+.

\begin{tabular}{|c|c|c|c|c|c|}
\hline \multirow[t]{2}{*}{ Network number } & \multicolumn{2}{|c|}{$\begin{array}{c}\text { Number } \\
\text { of }\end{array}$} & \multirow[t]{2}{*}{$\begin{array}{c}\text { Average } \\
\text { degree }\end{array}$} & \multirow{2}{*}{$\begin{array}{c}\text { Average } \\
\text { clustering } \\
\text { coefficient }\end{array}$} & \multirow{2}{*}{$\begin{array}{c}\text { Average } \\
\text { path } \\
\text { length }\end{array}$} \\
\hline & nodes & edges & & & \\
\hline$\# 107$ & 1034 & 26749 & 51.74 & 0.5264 & 2.95 \\
\hline \#1912 & 747 & 30025 & 80.39 & 0.6354 & 2.54 \\
\hline \#106186407539128840569 & 4289 & 246379 & 114.89 & 0.5759 & 2.57 \\
\hline \#110614416163543421878 & 338 & 10626 & 62.88 & 0.5418 & 1.98 \\
\hline
\end{tabular}

between them, and even a small shift of the market price can result in a drastic change of the demanded quantity. There are, however, some quantitative differences. In particular, strong WOM in the presence of adoption difficulty blocks the diffusion, but at a slightly higher market penetration level, as if the real network structure was somewhat more resistant to this influence. This issue definitely requires a more thorough investigation. Yet, the general guidelines for designing an adequate marketing strategy remain the same as put forward previously.
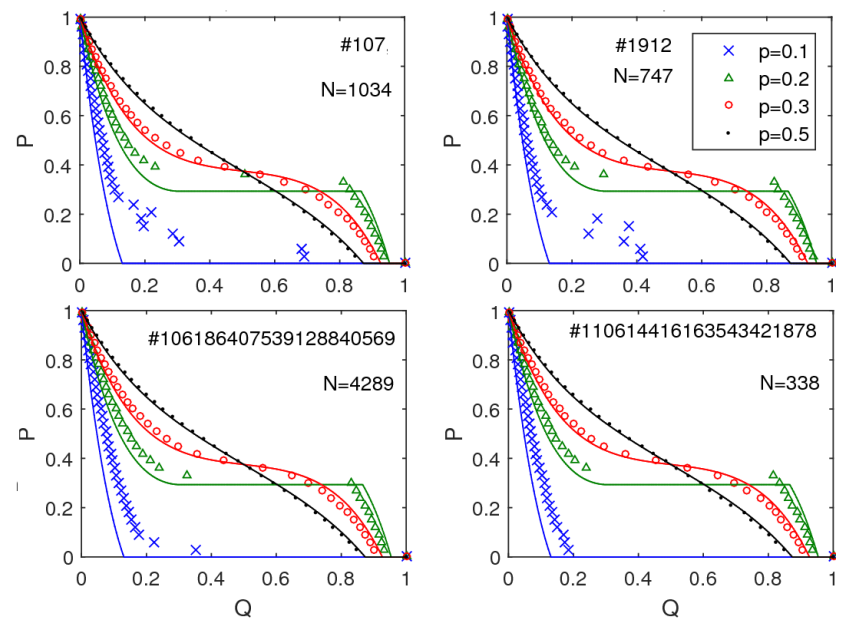

Fig. 3. Demand curves $P(Q)$ for models with different independence probabilities $p$, with a difficulty effect $(f=0.2)$ and without advertising $(h=0)$ considered on two Facebook (upper parts) and two Google+ (lower parts) networks. Monte Carlo simulation results for a complete graph consisting of $N=10^{4}$ spinsons are denoted by symbols, whereas the corresponding mean-field results for an infinite system by solid lines of the same color. Interestingly, the demand curves do not deviate very much from the curves obtained for complete graphs, at least for $p \geq 0.2$. Note that model parameters are the same as in the top right part in Fig. 1.

\section{Conclusions}

The micro-level interactions are typically transformed into the market behavior via the reservation prices. Although the concept of reservation prices is well known in the literature, it has not been explored very extensively in the context of agent-based models. There are only a few papers that relate agent opinions with reservation 
and market prices. However, most of them $[15,17,18]$ use the percolation approach, which is not consistent with the experimental data [19]. To our best knowledge, only one paper [5] uses an opinion formation framework, which has been found to be suitable for innovation diffusion modeling.

We have used the latter methodology for deriving and analyzing the demand curves of innovative goods. Our results show that social influence can significantly alter the impact of both adoption difficulty and advertising. The influence is not homogeneous and depends on the level of market prices. WOM associated with high market prices discourages diffusion and hence reduces the positive impact of advertising and strengthens the influence of the adoption difficulty. On the other hand, for low market prices, WOM boosts the diffusion and leads to higher demand. Moreover, it supports the positive response to advertising and weakens the effects of the adoption difficulty. This outcome may be considered as a recommendation for companies offering innovative products. Conditional on the targeted penetration level and the market price, the company should aim either at weakening or strengthening the WOM effect. By doing so, it can increase the demand and increase potential revenues.

Moreover, due to WOM, we could observe a switch from the low penetration into the high penetration regime. For strong social influence and low level of independence, there exists a price threshold, which separates these two regimes. For prices above the threshold, the diffusion is blocked and the penetration level remains very low. On the other hand, a small change of the price, which leads to the reduction of the price below the threshold, results in the rapid boost of the diffusion and a very high market share. Hence, the model predicts that there is a price level, for which the demand is extremely elastic and price miscalculation may have severe consequences for sales revenues and firm profits. Although a similar result was found by Zeppini and Frenken [15], two important details make our study particularly valuable. Firstly, in our setup, it is possible that the product will not diffuse even for very low market prices and will stay in the low penetration regime regardless of the price decisions of the producer. This may happen when the innovation is difficult to adopt and the social influence is strong. Secondly, our model predicts that it is possible to reach an almost perfect market penetration regime for moderate prices, which is not feasible in a percolation model.

\section{Acknowledgments}

We are grateful to an anonymous reviewer for inspiring comments. This work was supported by the National Science Center (NCN, Poland) through grant no. $2013 / 11 /$ B/HS4/01061.

\section{References}

[1] E.M. Rogers, Diffusion of Innovations, The Free Press, New York, NY 2003.

[2] J. Goldenberg, B. Libai, E. Muller, Int. J. Res. Marketing 27, 4 (2010).

[3] R. Peres, E. Muller, V. Mahajan, Int. J. Res. Marketing 27, 91 (2010).

[4] E. Kiesling, M. Güntherand, C. Stummer, L.M. Wakolbinger, Centr. Europ. J. Operat. Res. 20, 183 (2012).

[5] A. Kowalska-Pyzalska, K. Ćwik, A. Jędrzejewski, K. Sznajd-Weron, Acta Phys. Pol. A 129, 1055 (2016).

[6] P. Nyczka, K. Sznajd-Weron, J. Statist. Phys. 151, 174 (2013).

[7] H.R. Varian, Intermediate Microeconomics. A Modern Approach, 8th ed., W.W. Norton and Co., New York, NY 2010.

[8] P. Nyczka, K. Sznajd-Weron, J. Cisło, Phys. Rev. E 86, 011105 (2012).

[9] P.R. Nail, S.I. Di Domenico, G. MacDonald, Rev. Gen. Psychol. 17, 1 (2013).

[10] P.R. Nail, K. Sznajd-Weron, Acta Phys. Pol. A 129, (2016).

[11] F.G. Kaiser, K. Byrka, T. Hartig, Personal. Soc. Psychol. Rev. 14, 351 (2010).

[12] R. East, K. Hammond, W. Lomax, Int. J. Res. Market. 25, 215 (2008).

[13] A. Kowalska-Styczeń, K. Sznajd-Weron, Adv. Complex Syst. 15, 1250080 (2012).

[14] A. Campbell, Am. Econ. Rev. 103, 2466 (2013).

[15] P. Zeppini, K. Frenken, Working paper 38.15, University of Bath, Department of Economics, www. bath.ac.uk/economics/research/working-papers/ 2015-papers/networks-percolation-demand.pdf, 2015.

[16] K. Sznajd-Weron, J. Szwabiński, R. Weron, PLoS ONE 9, e112203 (2014).

[17] S. Cantono, G. Silverberg, Technolog. Forecast. Soc. Change 76, 487 (2009).

[18] M. Hohnisch, S. Pittnauer, D. Stauffer, Industr. Corpor. Change 17, 1001 (2008).

[19] C.H. Weiss, J. Poncela-Casasnovas, J.I. Glaser, A.R. Pah, S.D. Persell, D.W. Baker, R.G. Wunderink, L.A. Nunes Amaral, Phys. Rev. X 4, 041008 (2014). 